\title{
Correction: Cullin-5 neddylation-mediated NOXA degradation is enhanced by PRDX1 oligomers in colorectal cancer
}

Shoufang Xu, Yilei Ma, Qingchao Tong, Jun Yang, Jia Liu, Yanzhong Wang, Guoli Li, Jin Zeng, Sining Fang, Fengying Li, Xinyou Xie (D) and Jun Zhang (D)

Correction to: Cell Death and Disease https://doi.org/10.1038/s41419-021-03557-3 published online 12 March 2021

The original version of this article unfortunately contained a mistake in the legend of fig. 1B. The references in the sentence "The data sets were taken from TCGA ${ }^{35}$ and
Hong $^{36 "}$ were mis-marked and should be corrected to "The data sets were taken from TCGA ${ }^{25}$ and Hong ${ }^{26 "}$. The authors apologize for the mistake. The original article has been corrected.

Published online: 06 April 2021 